Relations industrielles

Industrial Relations

\title{
Simeon LARSON, Bruce NISSEN : Theories of the Labor Movement. Detroit, Wayne State University Press, 1987, 395 pp., ISBN 0-8143-1815-0
}

\section{Michel Audet}

\section{Volume 43, numéro 1, 1988}

URI : https://id.erudit.org/iderudit/050398ar

DOI : https://doi.org/10.7202/050398ar

Aller au sommaire du numéro

Éditeur(s)

Département des relations industrielles de l'Université Laval

ISSN

0034-379X (imprimé)

1703-8138 (numérique)

Découvrir la revue

Citer ce compte rendu

Audet, M. (1988). Compte rendu de [Simeon LARSON, Bruce NISSEN : Theories of the Labor Movement. Detroit, Wayne State University Press, 1987, 395 pp., ISBN 0-8143-1815-0]. Relations industrielles / Industrial Relations, 43(1), 199-200. https://doi.org/10.7202/050398ar

Tous droits réservés (C) Département des relations industrielles de l'Université Laval, 1988
Ce document est protégé par la loi sur le droit d'auteur. L’utilisation des services d’Érudit (y compris la reproduction) est assujettie à sa politique d'utilisation que vous pouvez consulter en ligne.

https://apropos.erudit.org/fr/usagers/politique-dutilisation/ 


\section{RECENSIONS}

\section{BOOK REVIEWS}

Theories of the Labor Movement, par Simeon Larson et Bruce Nissen, (Ed.), Détroit, Wayne State University Press, 1987, 395 pp., ISBN 0-8143-1815-0

L'enseignement des théories du mouvement ouvrier s'avère souvent une tâche complexe et difficile car les travaux à ce sujet sont dispersés dans une littérature relativement lourde, tant en termes de nombre qu'en termes de contenu.

C'est pour répondre à ce besoin que Larson et Nissen ont édité un recueil d'auteurs, regroupant plus d'une quarantaine des principaux théoriciens du mouvement ouvrier. On retrouve donc côte à côte des textes originaux aussi hétérogènes que ceux, par exemple, de Freeman et Medoff (What Do Unions Do), Karl Marx, André Gorz, John R. Commons, JeanPaul II (Laborem Exercens) et John Kenneth Galbraith.

En plus de recenser les principaux auteurs du mouvement ouvrier, ce volume a également l'avantage d'innover en classifiant les théories ou idéologies en sept groupes de base, selon le rôle social primaire qui est dévolu au mouvement ouvrier par chacun des théoriciens.

Le premier groupe considère le mouvement ouvrier comme un agent de révolution et se divise en trois sections, soit le marxisme (Marx, Engels, Hyman, etc.), le syndicalisme révolutionnaire (Trautmann, St-John, Haywood, etc.) et la «nouvelle gauche» (Gorz, Aronowitz). Ce premier groupe d'auteurs est le plus important dans ce volume, occupant près du tiers de l'ensemble.

Le deuxième groupe considère le mouvement ouvrier comme étant de l'ordre du syndicalisme d'affaires ou l'école du Wisconsin (Commons, Perlman, etc.). Le troisième groupe est celui de l'école des Webb (Sidney et Béatrice) qui considèrent le mouvement ouvrier comme un agent de réforme industriel. Le quatrième groupe, que l'on qualifie de réaction psychologique à l'industrialisation, se compose d'auteurs tels Parker, Hoxie et Veblen. Le cinquième groupe, qui qualifie le mouvement ouvrier de "force morale», est composé, entre autres, des encycliques de Léon XIII (Rerum Novarum), Pie XI (Quadragesimo Anno) et Jean-Paul II (Laborem Exercens). Le sixième groupe (Pernicious Monopoly) traite du mouvement ouvrier comme un monopole et ses principaux auteurs sont Henry Simons, Milton et Rose Friedman, de même que Freeman et Medoff. Finalement, le septième et dernier groupe est celui des tenants de la «société industrielle pluraliste», soit Kerr-Dunlop-Harbison-Myers (Industrialism and Industrial Man), Galbraith et Goldthorpe.

La présentation de ces sept groupes d'auteurs est précédée d'un texte de John Dunlop "The Development of Labor Organization: A Theoretical Framework» (1948), servant de cadre d'analyse à la définition scientifique d'une théorie du mouvement ouvrier, de même que d'une introduction qui met en garde le lecteur quant à la confusion «syndicalisme - mouvement ouvrier" et aux distinctions fondamentales à établir entre théorie et idéologie.

Outre le recensement des principaux auteurs du mouvement ouvrier et leur classification en fonction du «rôle social primaire», les principales forces de ce volume sont une considération élargie du «concept mouvement ouvrier», incluant le syndicalisme, une vision «pluraliste» 
du mouvement ouvrier faisant état aussi bien des tenants du syndicalisme d'affaires que du syndicalisme révolutionnaire et enfin, une attention portée à la fois sur des conceptions historiques et contemporaines (de Marx à Hyman ou Gorz, et des Webb à Kerr) du mouvement ouvrier.

Un tel volume gagnerait, par contre, à développer davantage et surtout en fonction de l'état actuel des connaissances scientifiques, un cadre d'analyse plus rationnel, plus opérationnel et plus contemporain que celui présenté par Dunlop dans la partie I. Un tel cadre d'analyse pourrait tenir compte, entre autres, du récent développement du concept «paradigme scientifique) et de son application aux sciences sociales.

La faiblesse la plus importante de ce volume est sans doute celle relative à son contenu, en ce sens que les deux éditeurs ont fait abstraction de toute la littérature européenne contemporaine sur le développement du mouvement ouvrier et du syndicalisme. Outre les auteurs français, dont certains ont été traduits en anglais (Touraine), les britanniques ont mené de nombreuses recherches à ce sujet, surtout sur le plan des idées. Citons par exemple un auteur comme Braverman (Labor Monopoly) qui a récemment laissé sa marque dans le domaine des relations industrielles.

Bref, bien que l'on puisse contester le contenu plutôt américain des théories du mouvement ouvrier que l'on retrouve dans ce volume, Larson et Nissen ont eu le mérite de recenser et de classifier, sous un même couvert, les diverses tendances en ce domaine et ce, dans leur contexte original et selon les ouvrages originaux de chaque époque.

Ce volume s'avère donc un outil pédagogique supplémentaire dans nos universités, afin de permettre aux professeurs et étudiants une meilleure compréhension des origines et du développement du mouvement ouvrier.

Michel AUDET

Université Laval

Strikes in Nova Scotia, 1970-1985, by C.H.J. Gilson, ed., Hantsport, N.S., Lancelot Press, 1986, 191 pp., ISBN 0-88999-314-9

It has often been remarked that each province has its own unique industrial relations system. One of the purposes of this modest collection of essays is to of fer insights into the pattern of industrial relations in contemporary Nova Scotia. This is done through a group of nine short papers, most of them prepared by student researchers at Saint Francis Xavier University. Each paper examines one recent labour dispute, providing an account of the causes, chronology and outcome. The research is generally based on daily newspapers, supplemented in several cases with interviews; there is little use of secondary literature on Nova Scotia history or on general issues in labour studies. The editor contributes an introduction and a conclusion which point out a number of common themes and suggest some of the weaknesses of the existing industrial relations system in the province.

The Canso fishermen's strike (1970-71) was perhaps the most significant of the nine disputes considered here. This was the strike which ended the legal fiction that fishermen were "coadventurers» - or even, the authors suggest, "management" (p. 19) who received a «salary» (p. 30) for their work! The strike has received considerable attention elsewhere, but this succinct treatment by Lorna Darrah and Rosalind Belland reminds us of the extraordinary 